MATEC Web of Conferences 11,01033 (2014)

DOI: $10.1051 /$ matecconf / 20141101033

(C) Owned by the authors, published by EDP Sciences, 2014

\title{
Valorisation et Recyclage des Déchets Plastiques dans le Béton
}

\author{
Benimam Samir ${ }^{1}$, DebiebFarid ${ }^{2}$, Bentchikou Mohamed $^{1}$ et Guendouz Mohamed ${ }^{2}$ \\ ${ }^{1}$ Laboratoire LBMPT, Université de Médéa, Algérie \\ ${ }^{2}$ Laboratoire LME (Ex. LPTRR), Université de Médéa, Algérie
}

\begin{abstract}
Résumé.La valorisation des déchets dans le génie civil est un secteur important dans la mesure où les produits que l'on souhaite obtenir ne sont pas soumis à des critères de qualité trop rigoureux. Le recyclage des déchets touche deux impacts très importants à savoir l'impact et l'impact économique. Donc plusieurs pays du monde, différents déchets sont utilisé dans le domaine de la construction et spécialement dans le ciment ou béton comme poudre, fibres ou agrégats. Ce travail s'intéresse à la valorisation d'un déchet qui est nuisible pour l'environnement vu son caractère encombrant et inesthétique il s'agit du déchet plastique. Trois types de déchets plastiques sont ajoutés dans le béton (sous forme de grains et fibres (ondulées et rectilignes). Les propriétés à l'état frais (maniabilité, air occlus et densité) et à l'état durci (résistance à la compression, à la traction, retrait et absorption d'eau) des différents bétons réalisés sont analysées et comparés par rapport leurs témoins respectifs. D'après les résultats expérimentaux on peut conclure que le renforcement de la matrice cimentaire avec des fibres plastiques ondulées montrent une nette amélioration de la résistance à la traction du béton ainsi qu'une diminution remarquable de sa capacité d'absorption de l'eau lorsqu'on utilise des grains plastiques.
\end{abstract}

\section{Introduction}

Depuis ces vingt dernières années, les déchets en matières plastiques représentent une part importante des déchets solides municipaux. De plus, ils posent un sérieux problème à cause de leur durée de vie et parce que ce sont des déchets voyants. Leur gestion est donc nécessaire que ce soit d'un point de vue environnemental, économique ou social. Les premiers procédés mis en place pour les traiter furent l'enfouissement et l'incinération. Toutefois, la croissance exponentielle de déchets d'emballages plastiques amène à prévoir d'autres filières de recyclage. Les matériaux de construction peuvent représenter un débouché intéressant.En effet, des études antérieures [1-4] ont montré qu'il était possible d'utiliser les déchets plastiques dans les bétons et mortiers et même d'autres types de déchets comme les granulats recyclés de démolition et/ou de construction $[5,6]$.

Notamment le polyéthylène téréphtalate (PET) recyclé, issu des emballages a été utilisé comme liant pour la production d'un matériau composite à hautes performances : le béton de polymère. Le plastique est transformé en présence de glycols, en résine polyester insaturée qui est ensuite mélangée avec du sable et des gravillons. Le béton de polymère obtenu est très résistant en compression et en flexion par rapport au béton de ciment Portland conventionnel [7]. Il présente aussi l'avantage d'atteindre $80 \%$ de ses résistances mécaniques dès les premiers jours de prise [3]. Mais il est sensible à la température [3]. D'autres auteurs ont aussi utilisé des déchets en PET mélangés à des déchets en polyéthylène haute densité (PEHD) comme granulats, en substitution partielle du sable (5 à $20 \%$ du volume total du sable) afin de les comparer aux fibres de verre généralement utilisées comme renfort de structure [8]. Leur étude a montré qu'une substitution volumique excédant $15 \%$ diminue les propriétés mécaniques des nouveaux composites par rapport au mortier de référence ne contenant pas de déchets.

Ce travail vise à étudier la possibilité d'utiliser des déchets de bouteilles plastiques (PET-polyéthylène téréphtalate) et des citernes (PEBD -polyéthylène bas densité) dans le béton sans autre transformation que le broyage, afin de minimiser le coût du matériau final. L'influence de la proportion des déchets utilisés sur les caractéristiques physiques et mécaniques du nouveau matériaua été étudiée et analysée.

\section{Protocol expérimental}

\subsection{Matériaux utilisés}

Un ciment Portland industriel de type CEM II/A 42.5R fabriqué en Algérie avec une surface spécifique de Blaine de $385 \mathrm{~m} 2 / \mathrm{kg}$, une masse volumique absolue de $4300 \mathrm{~kg} / \mathrm{m} 3$ et une résistance moyenne en compression à 
28 jours de $52.5 \mathrm{MPa}$ est utilisé pour tous les mélanges du mortier et béton. Ce ciment est sans ajouts spécifiques qui risqueraient de réagir avec les déchets plastiques, avec une faible chaleur en hydratation et une teneur limitée en sulfures.

Deux sables et deux graviers sont utilisés dans cette étude. Un sable fin roulé (SR 0/3) de HASSI BAHABAH de la région de DJEFA, située à $300 \mathrm{Km}$ au sud d'Alger et un autre sable grossier ( $\mathrm{SC} 0 / 5$ ) et deux graviers type concassés (G $0 / 3$ et $G$ 8/15) provenant de la station de concassage de MONT GORNO de la région de MEDEA située à $80 \mathrm{~km}$ au sud d'Alger en Algérie. Les caractéristiques physiques et mécaniques des sables sont résumées dans le tableau 1. La correction des deux sables suscités selon la méthode d'Abrams [9] nous a conduits à utiliser un troisième sable qu'on a appelé sable corrigé composé de $63.17 \%$ de sable roulé et de $63.83 \%$ de sable concassé.

Table 1.Caractéristiques des granulats

\begin{tabular}{|l|c|c|c|c|}
\hline \multirow{2}{*}{ Caractéristiques } & \multicolumn{2}{|c|}{ Sable } & \multicolumn{2}{c|}{ Gravier } \\
\cline { 2 - 5 } & $\begin{array}{c}\text { Roulé } \\
(\mathbf{0} / 3)\end{array}$ & $\begin{array}{c}\text { Concassée } \\
(\mathbf{0} / \mathbf{5})\end{array}$ & $\mathbf{3 / 8}$ & $\mathbf{8 / 1 5}$ \\
\hline $\mathrm{MF}$ & 1.00 & 3.35 & $/$ & $/$ \\
\hline $\mathrm{MVAb}(\mathrm{g} / \mathrm{cm} 3)$ & 2.48 & 2.54 & 2.50 & 2.53 \\
\hline $\mathrm{MVAp}(\mathrm{g} / \mathrm{cm} 3)$ & 1.42 & 1.49 & 1.29 & 1.27 \\
\hline ES $(\%)$ & 69.52 & 90.60 & $/$ & $/$ \\
\hline Abs. d'eau $(\%)$ & 1 & 1.8 & 0.70 & 0.80 \\
\hline Compacité & 56.35 & 57.36 & $/$ & $/$ \\
\hline Porosité & 43.65 & 42.63 & $/$ & $/$ \\
\hline Dureté & $/$ & $/$ & 24 & 26 \\
\hline
\end{tabular}

Aussi, Trois types de déchets plastiques (grains (GP), fibres (ondulées (FPO) et rectilignes (FPR)) [Fig. 1] sont ajouté dans le mortier et béton a différents pourcentages de substituions $(0 ; 5 ; 10 ; 15$ et $20 \%$ de grains plastiques par rapport à la masse du gravier) et d'incorporation $(0 ; 0.5 ; 1 ; 1.5$ et $2 \%$ de fibres plastiques (forme rectiligne ou ondulée) par rapport à la masse du ciment). L'eau de gâchage utilisé pour la confection des différents bétons est l'eau potable de distribution exempte d'impuretés.

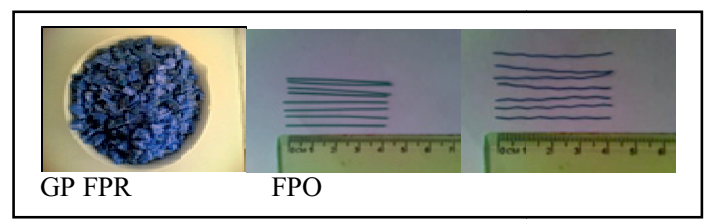

Fig. 1. Types de déchets plastiques utilisés

\subsection{Identification des bétons}

Pour le besoin de l'étude, en plus du béton de référence (Témoin), trois familles de béton sont réalisées :

MT : Béton témoin

BDGP : Béton de grains plastiques

BFPR : Béton de fibres plastiques rectilignes

BFPO : Béton de fibres plastiques ondulées

\subsection{Optimisation du mélange}

La composition du béton pour la confection des éprouvettes est basée sur la méthode de Dreux [9] qui a donné pour un dosage en ciment de $350 \mathrm{~kg} / \mathrm{m}^{3}$, un rapport gravier sur sable de 1,74 et un rapport d'eau sur ciment de 0,50 . Après démoulage des éprouvettes à $24 \mathrm{~h}$, la conservation se fait dans l'eau $\left(20^{\circ} \mathrm{C}\right)$ sauf pour les éprouvettes destinées aux essais de retrait et d'absorption capillaire qui sont conservés à l'air libre $\left(20\right.$ à $30^{\circ} \mathrm{C}$ et 50 à $60 \%$ d'humidité relative) à l'intérieur du laboratoire jusqu'au moment de l'essai.

L'eau efficace $\left(E_{\text {eff }}\right)$ est la quantité d'eau totale $\left(E_{\text {tot }}\right)$ dans le béton (eau de gâchage et eau apportée par les granulats) moins la quantité d'eau absorbée par les granulats $\left(\mathrm{E}_{\mathrm{gr}}\right)$.La quantité d'eau totale $\left(\mathrm{E}_{\mathrm{tot}}\right)$ utilisée pour le gâchage est la suivante (éq. 1) :

$\mathrm{E}_{\text {eff }}=\mathrm{E}_{\text {tot }}-\mathrm{E}_{\mathrm{gr}}=\mathrm{E}_{\mathrm{abs}}+\mathrm{E}_{\text {libre }}(1)$

$\mathrm{E}_{\text {tot }}$ : quantité d'eau totale utilisée pour le gâchage,

$\mathrm{E}_{\mathrm{gr}}$ : quantité d'eau initialement présente dans les granulats,

$\mathrm{E}_{\mathrm{abs}}$ : quantité d'eau absorbée par les granulats pendant le malaxage,

$\mathrm{E}_{\text {libre }}$ : quantité d'eau nécessaire au malaxage

\section{Résultats et discussions}

\subsection{Etat frais}

Pendant de la confection des éprouvettes, nous avons remarqué que les béton de déchets plastiques (BDP) sont peu compacts par apport au béton témoin (BT) et présentent une difficulté de mélange en fonction du pourcentage et du type d'ajout en déchets plastiques (grains ou fibres). Ceci confirme les résultats d'autres chercheurs [10-12]. Le temps d'écoulement des différents bétons est illustré dans les figures 2 et 3 . Les densités des bétons de déchets plastiques sont plus faibles $(8 \%$ cas BDGP et $4 \%$ cas BFP) que celui du BT.

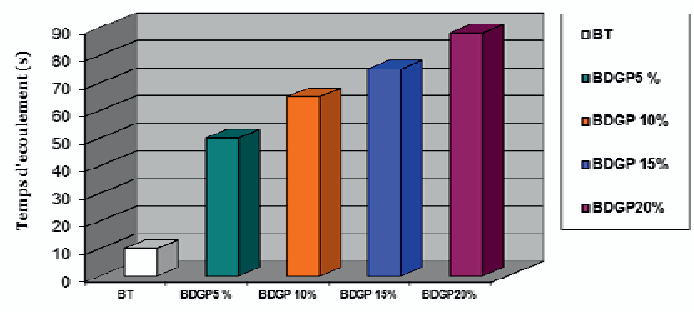

Fig. 2.Temps d'écoulement des bétons type BDGP

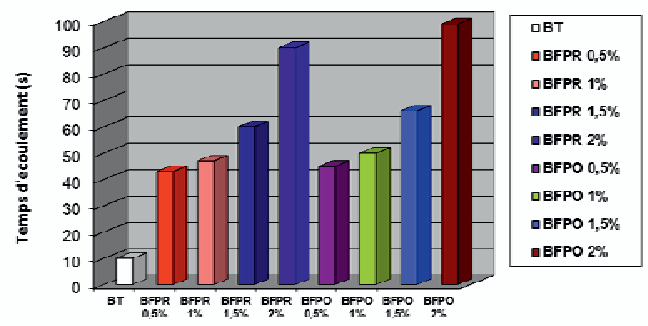

Fig. 3.Temps d'écoulement des bétonstype BFP 


\subsection{Etat durci}

Pour chaque essai, les résultats de la résistance mécanique du mortier est la moyenne de 3 mesures prisent sur 3 éprouvettes à l'âge de 3,7 et $28 \mathrm{j}$ et sont illustrés dans les Figures 4 et 5 suivantes :

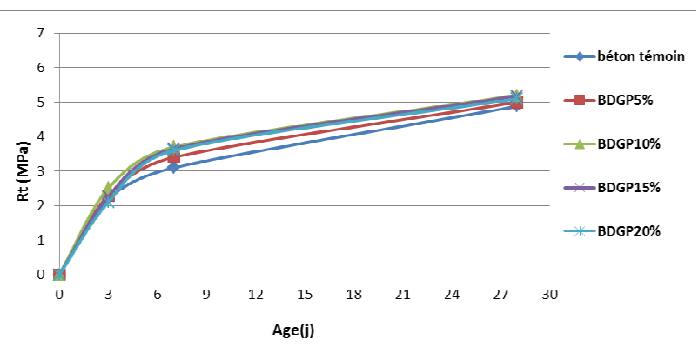

Fig. 4.Résistance à la traction des bétons type BDGP

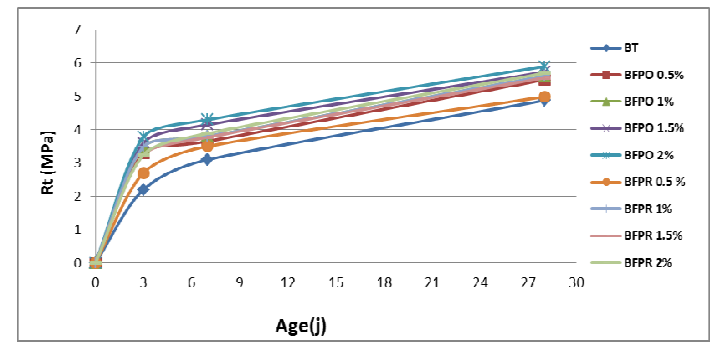

Fig. 5.Résistance à la traction des bétons type BFP

D'après les figures 4 et 5 on remarque que la cinétique de la résistance du béton à base de déchets plastiques est la comparableàcelle de béton témoin et que le béton gagne en résistance en fonction du pourcentage d'ajout en déchets plastiques. La résistance à la traction est améliorée d'une façon remarquable pour les bétons de fibres plastiques (ondulées et rectilignes) par rapport au béton de grains plastiques et par rapport au béton témoin. Ceci confirme les résultats de Al-Manaseer et al [10]. de Les fibres plastiques ondulées améliorent plus la résistance à la traction du béton (environ $25 \%$ de gain) que les fibres plastiques rectilignes. Ceci semble logique à cause de la forme ondulée qui augmente l'adhérence dans le squelette granulaire de la matrice cimentaire du béton.

Les figures 6,7 et 8 illustrent la cinétique d'absorption d'eau des différents mortiers confectionnés.

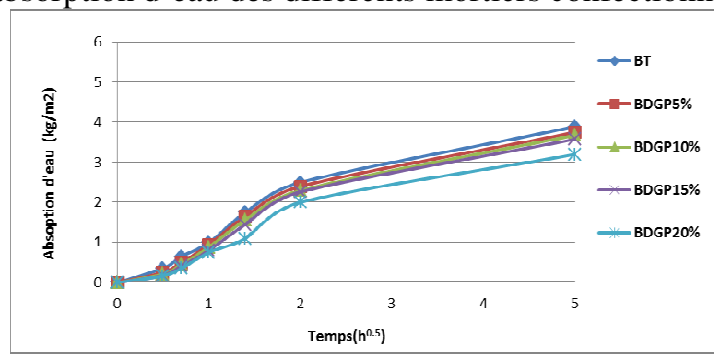

Fig. 6.Absorption d'eau des bétons type BDGP

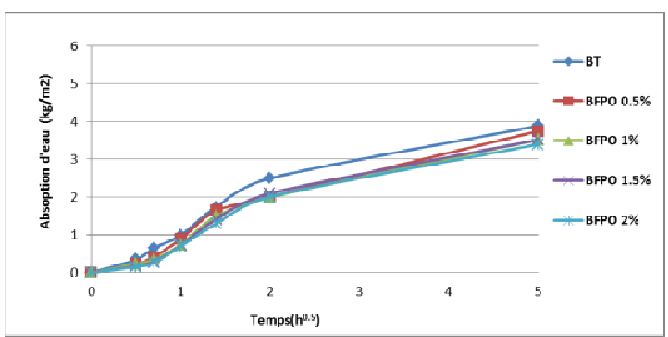

Fig. 7.Absorption d'eau des bétons type BFPO

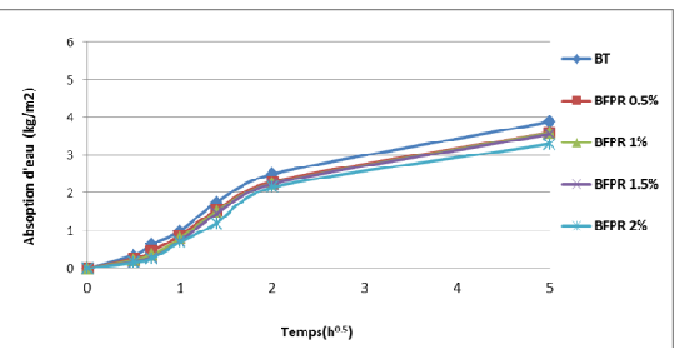

Fig. 8.Absorption d'eau des bétons type BFPR

D'après l'allure des figures 6,7 et 8 , Il est nettement visible que le processus d'absorption d'eau par les bétons de déchets plastiques est similaire à celui du béton naturel.Les fibres plastiques de forme ondulées contribuent encore d'avantage à la diminution de l'absorption d'eau des bétons (environ 9\%).

Les résultats de l'essai du retrait sur les différents bétons réalisés sont illustrés dans les figures 9,10 et 11 suivantes.

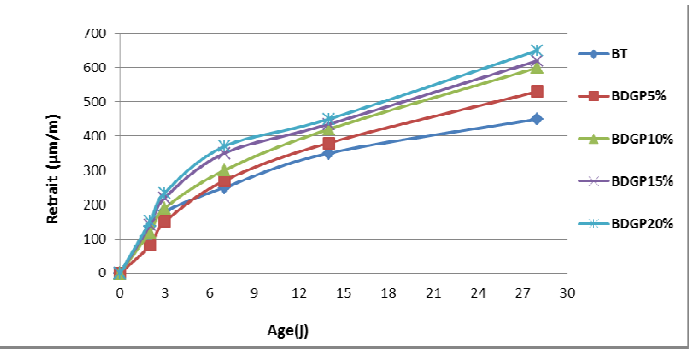

Fig. 9.Retrait des bétons type BDGP

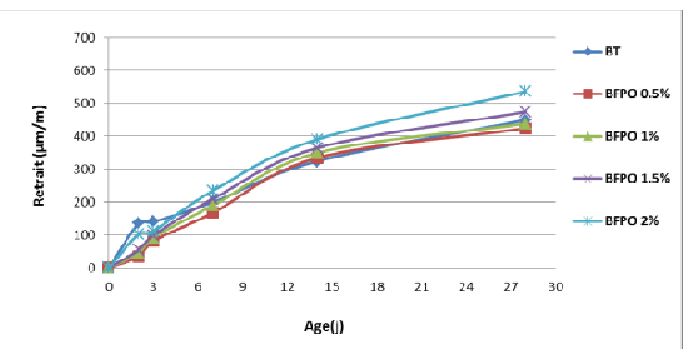

Fig. 10.Retrait des bétons type BFPO

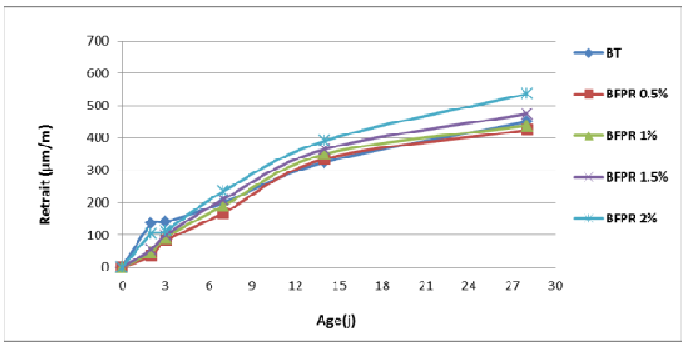

Fig. 11. Retrait des bétons type BFPR 
La figure 9 montre que tous les bétons à base de déchets plastiquesont des retraitsplus grands par rapport au béton témoin, et on remarque que plus le pourcentage augmente plus le retrait augmente. Contrairement aux grains plastiques, les fibres plastiques (de forme ondulée ou rectiligne) contribuent au retardement (d'environ -8\%) du retrait du béton jusqu'au pourcentage d'ajout de $1 \%$ en fibres et au-delà le retrait reprend sa croissance (une augmentation de+13\%) pour un pourcentage de $2 \%$ d'ajout en fibres.

\section{Conclusions}

L'objectif de ce travail est de valoriser les déchets plastiques et étudier leurs effets sur le comportement du béton. Sur la base des résultats obtenues on conclut que :

- le béton de déchets plastiques est peu compact par apport au béton naturel et présente une difficulté de mélange et de mise en œuvre en fonction du pourcentage et du type d'ajout en déchets plastiques (grains ou fibres).

- Le béton armé de fibres plastiques (surtout en forme ondulée) résiste mieux à la flexion par la traction en comparaison au béton naturel. Néanmoins un risque de retrait supplémentaire est possible pour un dosage élevé en pourcentage de fibres, c'est pourquoi un pourcentage de $1 \%$ en fibres à ne pas dépasser est conseillé.

\section{Références}

1. V. T. Breslin, U. Senturk, C. C. Berndt, Long-term engineering properties of recycled plastic lumber in pier construction. Resources, Conservation and Recycling 23, 243-258 (1998)

2. K.Flaga, Advanced in materials applied in civil engineering. Journal of Materials Processing Technology 106,173-183 (2000)

3. K. S. Rebeiz, Time-Temperature properties of polymer concrete using recycled PET. Cement and Concrete Composites 17, 119-124 (1995)

4. S. Hinislioglu, E. Agar, Use of high density polyethylene as bitumen modifier in asphalt concrete mix.Materials Letters 28, 267-271(2004)

5. F. Debieb , S. Kenai. The use of fine and coarse crushed bricks as aggregates in concrete. Constr Build Mater 22(5):886-93. (2008)

6. F. Debieb, L. Courard, S. Kenai, R. Degeimbre. Mechanical and durability properties of concrete using contaminated recycled aggregates. Cem Con Comp 32 (8):421-426 (2010)

7. T. D. Sam, M. N. Tam,Polymer concrete based on recycled polyethylene terephtalate (PET). NOCMAT/3.Vietnam International Conference on Non-Conventional Material and Technologies, 226$228(2002)$

8. J. VVaverka,An analysis of reinforced concrete composites utilizing recycled polyethylene terephthalate thermoplastic. Doctoral Thesis, University of Northern Iowa, LD2585.2.V39 (1991)

9. G. Dreux, J. Festa, Nouveau guide du béton. Eightedition. Paris: Eyrolles (1998)
10. A.A. Al-Manaseer, T. R. Dalal.. Concrete containing plastic aggregates. Concrete International, 47- 52 (1997)

11. K. Hannawi, S. Kamali-Bernard, W. Prince. Physical and mechanical properties of mortars containing PET and PC waste aggregates. Waste Management 30:2312-20 (2010)

12. S. Kim, N. Yi, H. Kim, J. Kim, Y. Song. Material and structural performance evaluation of recycled PET fiber reinforced concrete. Cem Conc Comp 32:232-40 (2010) 